\title{
Spin Sum Rules and Polarizabilities: Results from Jefferson Lab
}

\author{
Jian-ping Chen \\ Thomas Jefferson National Accelerator Facility \\ 12000 Jefferson Ave., Newport News, Virginia 23606, USA \\ *E-mail: jpchen@jlab.org
}

\begin{abstract}
The nucleon spin structure has been an active, exciting and intriguing subject of interest for the last three decades. Recent experimental data on nucleon spin structure at low to intermediate momentum transfers provide new information in the confinement regime and the transition region from the confinement regime to the asymptotic freedom regime. New insight is gained by exploring moments of spin structure functions and their corresponding sum rules (i.e. the generalized Gerasimov-Drell-Hearn, Burkhardt-Cottingham and Bjorken). The Burkhardt-Cottingham sum rule is verified to good accuracy. The spin structure moments data are compared with Chiral Perturbation Theory calculations at low momentum transfers. It is found that chiral perturbation calculations agree reasonably well with the first moment of the spin structure function $g_{1}$ at momentum transfer of 0.05 to $0.1 \mathrm{GeV}^{2}$ but fail to reproduce the neutron data in the case of the generalized polarizability $\delta_{L T}$ (the $\delta_{L T}$ puzzle). New data have been taken on the neutron $\left({ }^{3} \mathrm{He}\right)$, the proton and the deuteron at very low $Q^{2}$ down to $0.02 \mathrm{GeV}^{2}$. They will provide benchmark tests of Chiral dynamics in the kinematic region where the Chiral Perturbation theory is expected to work.
\end{abstract}

Keywords: Nucleon; Spin; Sum Rule; Polarizability; Moment; Jefferson Lab.

\section{Introduction}

In the last twenty-five years the study of the spin structure of the nucleon led to a very productive experimental and theoretical activity with exciting results and new challenges. ${ }^{1}$ This investigation has included a variety of aspects, such as testing QCD in its perturbative regime via spin sum rules (like the Bjorken sum rule ${ }^{2}$ ) and understanding how the spin of the nucleon is built from the intrinsic degrees of freedom of the theory, quarks and gluons. Recently, results from a new generation of experiments performed at Jefferson Lab seeking to probe the theory in its non-perturbative and transition regimes have reached a mature state. The low momentum-transfer 
results offer insight in a region known for the collective behavior of the nucleon constituents and their interactions. Furthermore, distinct features seen in the nucleon response to the electromagnetic probe, depending on the resolution of the probe, point clearly to different regimes of description, i.e. a scaling regime where quark-gluon correlations are suppressed versus a coherent regime where long-range interactions give rise to the static properties of the nucleon.

In this talk we describe an investigation ${ }^{4-9}$ of the spin structure of the nucleon through the measurement of the helicity-dependent photoabsorption cross sections or asymmetries using virtual photons across a wide resolution spectrum. These observables are used to extract the spin structure functions $g_{1}$ and $g_{2}$ and evaluate their moments. These moments are powerful tools to test QCD sum rules and Chiral Perturbation Theory calculations.

\section{Sum rules and Moments}

Sum rules involving the spin structure of the nucleon offer an important opportunity to study QCD. In recent years the Bjorken sum rule at large $Q^{2}$ (4-momentum transfer squared) and the Gerasimov, Drell and Hearn (GDH) sum rule ${ }^{10}$ at $Q^{2}=0$ have attracted large experimental and theoretical efforts ${ }^{3}$ that have provided us with rich information. Another type of sum rules, such as the generalized GDH sum rule ${ }^{11}$ or the polarizability sum rules, ${ }^{12}$ relate the moments of the spin structure functions to real or virtual Compton amplitudes, which can be calculated theoretically. These sum rules are based on "unsubtracted" dispersion relations and the optical theorem.

Considering the forward spin-flip doubly-virtual Compton scattering (VVCS) amplitude $g_{T T}$ and assuming it has an appropriate convergence behavior at high energy, an unsubtracted dispersion relation leads to the following equation for ${ }^{9,12} g_{T T}$ :

$$
\operatorname{Re}\left[g_{T T}\left(\nu, Q^{2}\right)-g_{T T}^{\text {pole }}\left(\nu, Q^{2}\right)\right]=\left(\frac{\nu}{2 \pi^{2}}\right) \mathcal{P} \int_{\nu_{0}}^{\infty} \frac{K\left(\nu^{\prime}, Q^{2}\right) \sigma_{T T}\left(\nu^{\prime}, Q^{2}\right)}{\nu^{\prime 2}-\nu^{2}} d \nu^{\prime},
$$

where $g_{T T}^{\text {pole }}$ is the nucleon pole (elastic) contribution, $\mathcal{P}$ denotes the principal value integral and $K$ is the virtual photon flux factor. The lower limit of the integration $\nu_{0}$ is the pion-production threshold on the nucleon. A low-energy expansion gives:

$$
\operatorname{Re}\left[g_{T T}\left(\nu, Q^{2}\right)-g_{T T}^{p o l e}\left(\nu, Q^{2}\right)\right]=\left(\frac{2 \alpha}{M^{2}}\right) I_{T T}\left(Q^{2}\right) \nu+\gamma_{0}\left(Q^{2}\right) \nu^{3}+O\left(\nu^{5}\right) .
$$


Combining Eqs. (1) and (2), the $O(\nu)$ term yields a sum rule for the generalized GDH integral: ${ }^{3,11}$

$$
\begin{aligned}
I_{T T}\left(Q^{2}\right) & =\frac{M^{2}}{4 \pi^{2} \alpha} \int_{\nu_{0}}^{\infty} \frac{K\left(\nu, Q^{2}\right)}{\nu} \frac{\sigma_{T T}}{\nu} d \nu \\
= & \frac{2 M^{2}}{Q^{2}} \int_{0}^{x_{0}}\left[g_{1}\left(x, Q^{2}\right)-\frac{4 M^{2}}{Q^{2}} x^{2} g_{2}\left(x, Q^{2}\right)\right] d x .
\end{aligned}
$$

The low-energy theorem relates $\mathrm{I}(0)$ to the anomalous magnetic moment of the nucleon, $\kappa$, and Eq. (3) becomes the original GDH sum rule: ${ }^{10}$

$$
I(0)=\int_{\nu_{0}}^{\infty} \frac{\sigma_{1 / 2}(\nu)-\sigma_{3 / 2}(\nu)}{\nu} d \nu=-\frac{2 \pi^{2} \alpha \kappa^{2}}{M^{2}},
$$

where $2 \sigma_{T T} \equiv \sigma_{1 / 2}-\sigma_{3 / 2}$. The $O\left(\nu^{3}\right)$ term yields a sum rule for the generalized forward spin polarizability: ${ }^{12}$

$$
\begin{aligned}
\gamma_{T T}\left(Q^{2}\right)=\left(\frac{1}{2 \pi^{2}}\right) \int_{\nu_{0}}^{\infty} \frac{K\left(\nu, Q^{2}\right)}{\nu} \frac{\sigma_{T T}\left(\nu, Q^{2}\right)}{\nu^{3}} d \nu \\
=\frac{16 \alpha M^{2}}{Q^{6}} \int_{0}^{x_{0}} x^{2}\left[g_{1}\left(x, Q^{2}\right)-\frac{4 M^{2}}{Q^{2}} x^{2} g_{2}\left(x, Q^{2}\right)\right] d x .
\end{aligned}
$$

Considering the longitudinal-transverse interference amplitude $g_{L T}$, the $O\left(\nu^{2}\right)$ term leads to the generalized longitudinal-transverse polarizability: ${ }^{12}$

$$
\begin{aligned}
\delta_{L T}\left(Q^{2}\right) & =\left(\frac{1}{2 \pi^{2}}\right) \int_{\nu_{0}}^{\infty} \frac{K\left(\nu, Q^{2}\right)}{\nu} \frac{\sigma_{L T}\left(\nu, Q^{2}\right)}{Q \nu^{2}} d \nu \\
= & \frac{16 \alpha M^{2}}{Q^{6}} \int_{0}^{x_{0}} x^{2}\left[g_{1}\left(x, Q^{2}\right)+g_{2}\left(x, Q^{2}\right)\right] d x .
\end{aligned}
$$

Alternatively, we can consider the covariant spin-dependent VVCS amplitudes $S_{1}$ and $S_{2}$, which are related to the spin-flip amplitudes $g_{T T}$ and $g_{L T}$. The unsubtracted dispersion relations for $S_{2}$ and $\nu S_{2}$ lead to a "superconvergence relation" that is valid for any value of $Q^{2}$,

$$
\int_{0}^{1} g_{2}\left(x, Q^{2}\right) d x=0
$$

which is the Burkhardt-Cottingham (BC) sum rule. ${ }^{13}$

At high $Q^{2}$, the $\mathrm{OPE}^{14}$ for the VVCS amplitude leads to the twist expansion. The leading-twist (twist-2) component can be decomposed into flavor triplet $\left(g_{A}\right)$, octet $\left(a_{8}\right)$ and singlet $(\Delta \Sigma)$ axial charges. The difference between the proton and the neutron gives the flavor non-singlet term:

$$
\Gamma_{1}^{p}\left(Q^{2}\right)-\Gamma_{1}^{n}\left(Q^{2}\right)=\frac{1}{6} g_{A}+O\left(\alpha_{s}\right)+O\left(1 / Q^{2}\right),
$$


which becomes the Bjorken sum rule at the $Q^{2} \rightarrow \infty$ limit.

The leading-twist part provides information on the polarized parton distributions. The higher-twist parts are related to quark-gluon interations or correlations. Of particular interest is the twist- 3 component, $d_{2}$, which is related to the second moment of the twist-3 part of $g_{1}$ and $g_{2}$ :

$$
\begin{aligned}
d_{2}\left(Q^{2}\right) & =\int_{0}^{1} d x x^{2}\left(2 g_{1}\left(x, Q^{2}\right)+3 g_{2}\left(x, Q^{2}\right)\right) \\
= & 3 \int_{0}^{1} d x x^{2}\left(g_{2}\left(x, Q^{2}\right)-g_{2}^{W W}\left(x, Q^{2}\right)\right),
\end{aligned}
$$

where $g_{2}^{W W}$ is the twist-2 part of $g_{2}$ as derived by Wandzura and Wilczek ${ }^{15}$

$$
g_{2}^{W W}\left(x, Q^{2}\right)=g_{1}\left(x, Q^{2}\right)+\int_{x}^{1} d y \frac{g_{1}\left(y, Q^{2}\right)}{y} .
$$

$d_{2}$ is related to the color electric and magnetic polarizabilities, which describe the response of the collective color electric and magnetic fields to the spin of the nucleon. ${ }^{14}$

\section{Description of the JLab experiments}

The inclusive experiments described here took place in JLab Halls $\mathrm{A}^{18}$ and B. ${ }^{19}$ The accelerator produces a polarized electron beam of energy up to 6 $\mathrm{GeV}$.

A polarized high-pressure ( $\sim 12$ atm.) gaseous ${ }^{3} \mathrm{He}$ target was used as an effective polarized neutron target in the experiments performed in Hall A. The average target polarization, monitored by NMR and EPR techniques, was $0.4 \pm 0.014$ and its direction could be oriented longitudinal or transverse to the beam direction. The measurement of cross sections in the two orthogonal directions allowed a direct extraction of $g_{1}^{3 \mathrm{He}}$ and $g_{2}^{3 \mathrm{He}}$, or equivalently $\sigma_{T T}$ and $\sigma_{L T}$.

The scattered electrons were detected by two High Resolution Spectrometers (HRS) with the associated detector package. The high luminosity of $10^{36} \mathrm{~cm}^{-2} \mathrm{~s}^{-1}$ allowed for statistically accurate data.

The spin structure functions $g_{1}^{n}$ and $g_{2}^{n}$ are extracted using polarized cross-section differences. Electromagnetic radiative corrections were performed. Nuclear corrections are applied via a PWIA-based model..$^{20}$ To form the moments, the integrands (e.g. $\sigma_{T T}$ or $g_{1}$ ) were determined from the measured points by interpolation. To complete the moments for the unmeasured high-energy region, the Bianchi and Thomas parameterization ${ }^{22}$ was used for $4<W^{2}<1000 \mathrm{GeV}^{2}$ and a Regge-type parameterization was 
used for $W^{2}>1000 \mathrm{GeV}^{2}$. Polarized solid ${ }^{15} \mathrm{NH}_{3}$ and ${ }^{15} \mathrm{ND}_{3}$ targets using dynamic nuclear polarization were used in Hall B. The CEBAF Large Acceptance Spectrometer (CLAS) in Hall B, which has a large angular $(2.5 \pi$ sr) and momentum acceptance, was used to detect scattered electrons. The spin structure functions were extracted using asymmetry measurements together with the world unpolarized structure function fits. ${ }^{21}$ Radiative corrections were applied.

\section{Recent results from Jefferson Lab}

\subsection{Results of the generalized GDH sum and $B C$ sum for ${ }^{3} \mathrm{He}$ and the neutron}

Fig. 1 shows the extended GDH integrals $I\left(Q^{2}\right)$ (open circles) for ${ }^{3} \mathrm{He}$ (preliminary) (upper-left) and for the neutron (upper-right), which were extracted from Hall A experiment E94-010 ${ }^{4}$, from break-up threshold for ${ }^{3} \mathrm{He}$ (from pion threshold for the neutron) to $W=2 \mathrm{GeV}$. The uncertainties, when visible, represent statistics only; the systematics are shown by the grey band. The solid squares include an estimate of the unmeasured high-energy part. The corresponding uncertainty is included in the systematic uncertainty band. The preliminary ${ }^{3} \mathrm{He}$ results rise with decreasing $Q^{2}$. Since the GDH sum rule at $Q^{2}=0$ predicts a large negative value, a drastic turn around should happen at $Q^{2}$ lower than $0.1 \mathrm{GeV}^{2}$. A simple model using MAID $^{3}$ plus quasielastic contributions indeed shows the expected turn around. The data at low $Q^{2}$ should be a good test ground for few-body Chiral Pertubation Theory Calculations.

The neutron results indicate a smooth variation of $I\left(Q^{2}\right)$ to increasingly negative values as $Q^{2}$ varies from $0.9 \mathrm{GeV}^{2}$ towards zero. The data are more negative than the MAID model calculation. ${ }^{3}$ Since the calculation only includes contributions to $I\left(Q^{2}\right)$ for $W \leq 2 \mathrm{GeV}$, it should be compared with the open circles. The GDH sum rule prediction, $I(0)=-232.8 \mu \mathrm{b}$, is indicated in Fig. 1, along with extensions to $Q^{2}>0$ using two nextto-leading order $\chi \mathrm{PT}$ calculations, one using the Heavy Baryon approximation $(\mathrm{HB} \chi \mathrm{PT})^{23}$ (dotted line) and the other Relativistic Baryon $\chi \mathrm{PT}$ $(\mathrm{RB} \chi \mathrm{PT})^{24}$ (dot-dashed line). Shown with a grey band is $\mathrm{RB} \chi \mathrm{PT}$ including resonance effects, ${ }^{24}$ which have an associated large uncertainty due to the resonance parameters used.

The capability of transverse polarization of the Hall $\mathrm{A}^{3} \mathrm{He}$ target allows precise measuremetns of $g_{2}$. The integral of $\Gamma_{2}^{3} \mathrm{He}$ (preliminary) and $\Gamma_{2}^{n}$ is plotted in the lower-left and lower-right panels of Fig. 1 in the measured 

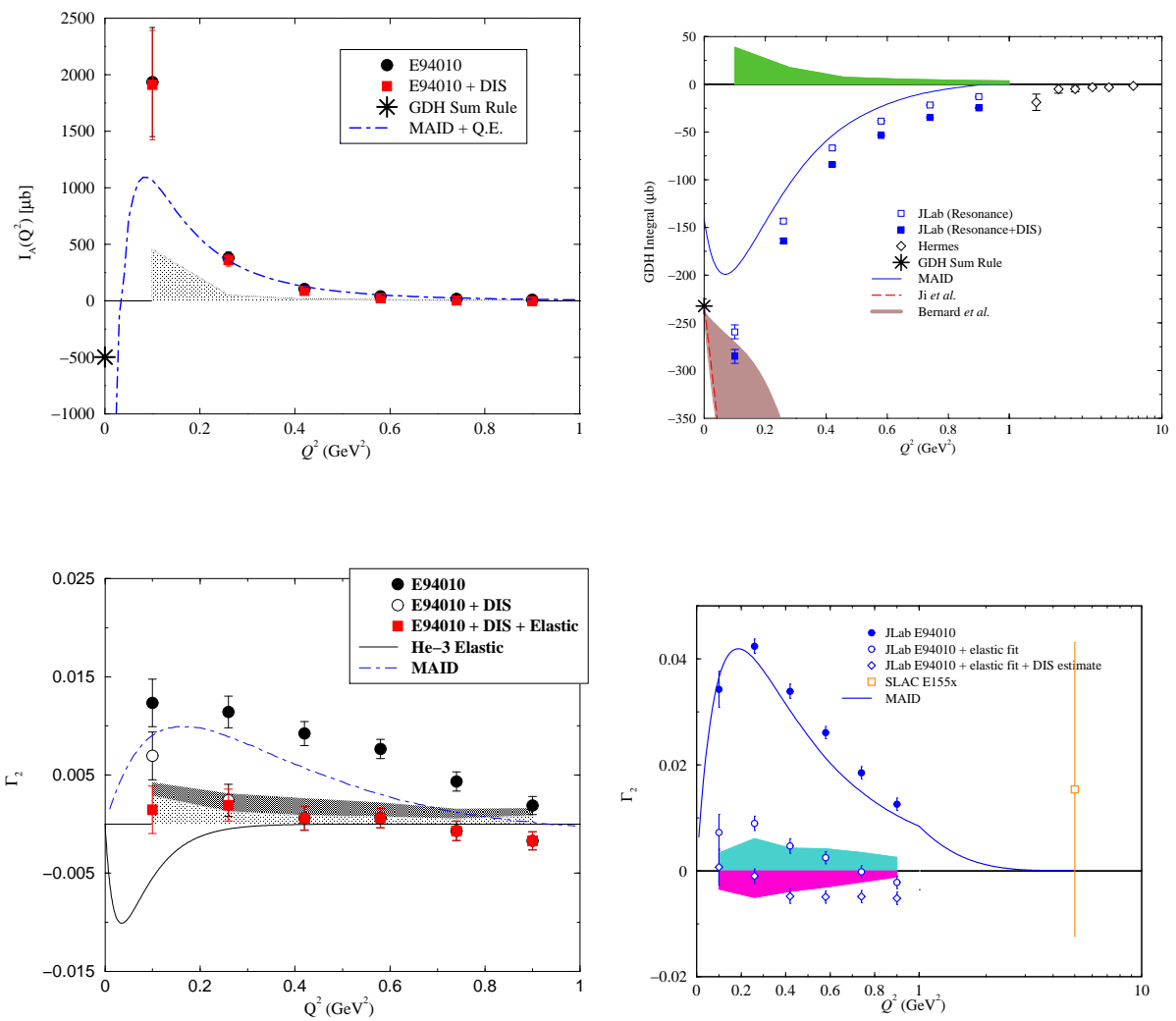

Fig. 1. Results of GDH sum $I\left(Q^{2}\right)$ and BC sum $\Gamma_{2}\left(Q^{2}\right)$ for ${ }^{3} \mathrm{He}$ (preliminary) and the neutron. The ${ }^{3} \mathrm{He}$ GDH results are compared with the MAID model plus quasielastic contribution. The neutron GDH results are compared with $\chi \mathrm{PT}$ calculations of ref. ${ }^{23}$ (dotted line) and ref. ${ }^{24}$ (dot-dashed line). The MAID model calculation of ref., ${ }^{3}$ is represented by a solid line. Data from HERMES ${ }^{16}$ are also shown. The BC sum results (resonance only) are compared with MAID model calculations.

region (solid circles) and open circles show the results after adding an estimated DIS contribution for ${ }^{3} \mathrm{He}$ (elastic contribution for the neutron). The solid squares (open diamonds) correspond to the results obtained after adding the elastic contributions for ${ }^{3} \mathrm{He}$, (adding an estimated DIS contribution assuming $g_{2}=g_{2}^{W W}$ for the neutron). The MAID estimate agrees with the general trend but slightly lower than the resonance data. The two bands correspond to the experimental systematic errors and the estimate of the systematic error for the low- $x$ extrapolation. The total results are consistent with the BC sum rule. The SLAC E155x collaboration ${ }^{17}$ previously 
reported a neutron result at high $Q^{2}$ (open square), which is consistent with zero but with a rather large error bar. On the other hand, the SLAC proton result was reported to deviate from the BC sum rule by 3 standard deviations.

\subsection{First moments of $g_{1}$ and the Bjorken sum}

The preliminary results from Hall B EG1b ${ }^{8}$ experiment on $\bar{\Gamma}_{1}\left(Q^{2}\right)$ at low to moderate $Q^{2}$ are shown together with published results from Hall $\mathrm{A}^{4}$ and Hall B eg $1 a^{5,6}$ in Fig. 2 along with the data from SLAC ${ }^{17}$ and HERMES. ${ }^{16}$ The new results are in good agreement with the published data. The inner uncertainty indicates the statistical uncertainty while the outer one is the quadratic sum of the statistical and systematic uncertainties.

At $Q^{2}=0$, the GDH sum rule predicts the slopes of moments (dotted lines). The deviation from the slopes at low $Q^{2}$ can be calculated with $\chi \mathrm{PT}$. We show results of calculations by Ji et al. ${ }^{23}$ using $\mathrm{HB} \chi \mathrm{PT}$ and by Bernard et al. with and without ${ }^{24}$ the inclusion of vector mesons and $\Delta$ degrees of freedom. The calculations are in reasonable agreements with the data at the lowest $Q^{2}$ settings of $0.05-0.1 \mathrm{GeV}^{2}$. At moderate and large $Q^{2}$ data are compared with two model calculations ${ }^{25,26}$. Both models agree well with the data. The leading-twist pQCD evolution is shown by the grey band. It tracks the data down to surprisingly low $Q^{2}$, which indicates an overall suppression of higher-twist effects.

\subsection{Spin Polarizabilities: $\gamma_{0}, \delta_{L T}$ and $d_{2}$ for the neutron}

The generalized spin polarizabilities provide benchmark tests of $\chi \mathrm{PT}$ calculations at low $Q^{2}$. Since the generalized polarizabilities have an extra $1 / \nu^{2}$ weighting compared to the first moments (GDH sum or $I_{L T}$ ), these integrals have less contributions from the large- $\nu$ region and converge much faster, which minimizes the uncertainty due to the unmeasured region at large $\nu$.

At low $Q^{2}$, the generalized polarizabilities have been evaluated with next-to-leading order $\chi \mathrm{PT}$ calculations. ${ }^{24,32}$ One issue in the $\chi \mathrm{PT}$ calculations is how to properly include the nucleon resonance contributions, especially the $\Delta$ resonance. As was pointed out in Refs. ${ }^{24,32}$, while $\gamma_{0}$ is sensitive to resonances, $\delta_{L T}$ is insensitive to the $\Delta$ resonance. Measurements of the generalized spin polarizabilities are an important step in understanding the dynamics of QCD in the chiral perturbation region.

The first results for the neutron generalized forward spin polarizabilities 

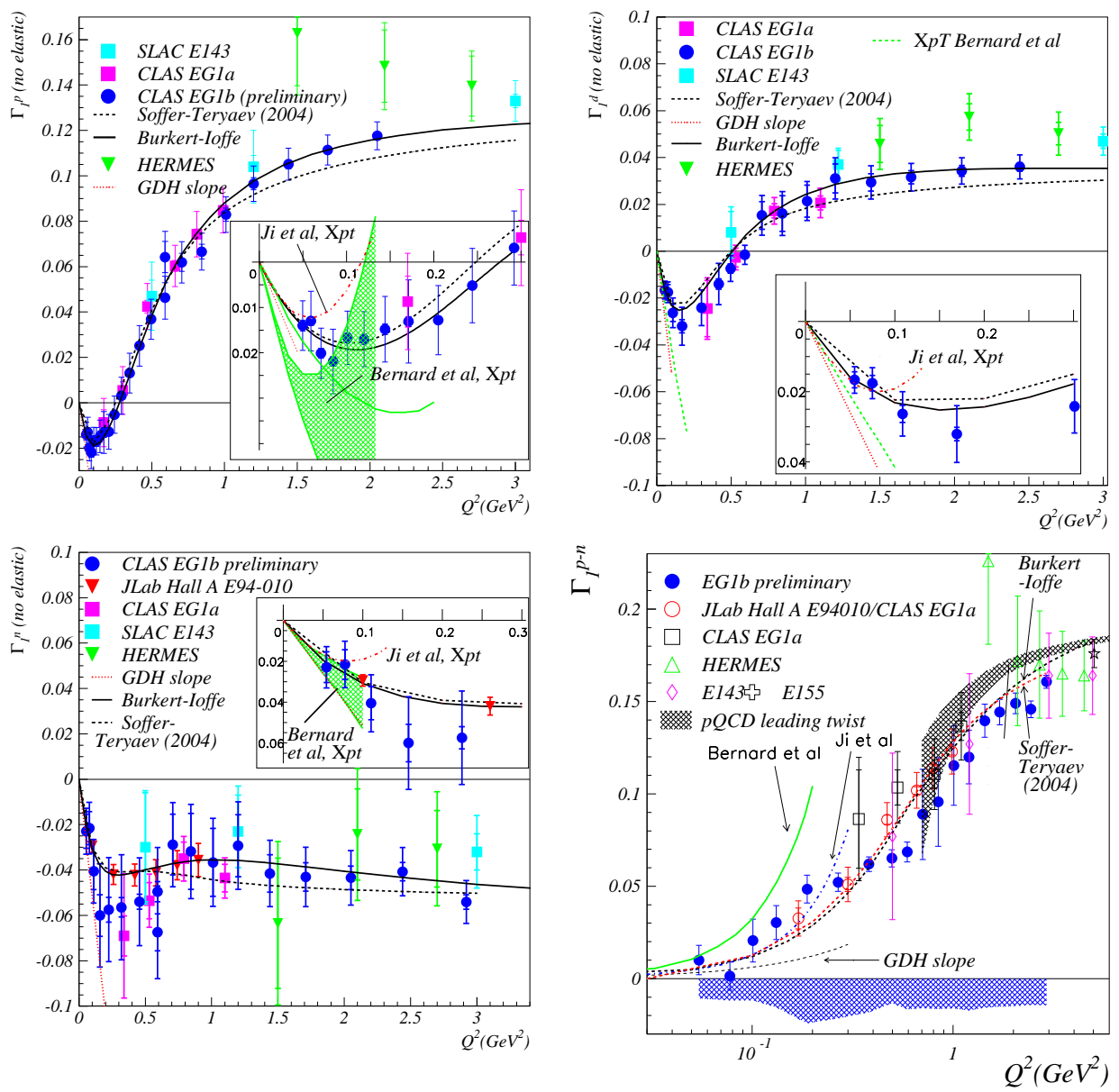

Fig. 2. Preliminary results of $\Gamma_{1}\left(Q^{2}\right)$ from CLAS eg $1 \mathrm{~b}^{8}$ for $\mathrm{p}, \mathrm{d}, \mathrm{n}$ and $\mathrm{p}-\mathrm{n}$, together with the published results from Hall $\mathrm{A}^{4}$ and CLAS eg $1 \mathrm{a}^{5,6}$. The slopes at $Q^{2}=0$ predicted by the GDH sum rule are given by the dotted lines. The MAID model predictions that include only resonance contributions are shown by the full lines while the dashed (dotdashed) lines are the predictions from the Soffer-Teryaev (Burkert-Ioffe) model. The leading twist $Q^{2}$-evolution of the moments is given by the grey band. The insets show comparisons with $\chi \mathrm{PT}$ calculatiosns. The full lines (bands) at low $Q^{2}$ are the next-toleading order $\chi \mathrm{PT}$ predictions by Ji et al. (Bernard et al.).

$\gamma_{0}\left(Q^{2}\right)$ and $\delta_{L T}\left(Q^{2}\right)$ were obtained at Jefferson Lab Hall A. ${ }^{4}$ The results for $\gamma_{0}\left(Q^{2}\right)$ are shown in the top-left panel of Fig. 3. The statistical uncertainties are smaller than the size of the symbols. The data are compared with a next-to-leading order $\left(O\left(p^{4}\right)\right) \mathrm{HB} \chi \mathrm{PT}$ calculation, ${ }^{32}$ a next-to-leading order $\mathrm{RB} \chi \mathrm{PT}$ calculation and the same calculation explicitly including both the $\Delta$ resonance and vector meson contributions. ${ }^{24}$ Predictions from the 


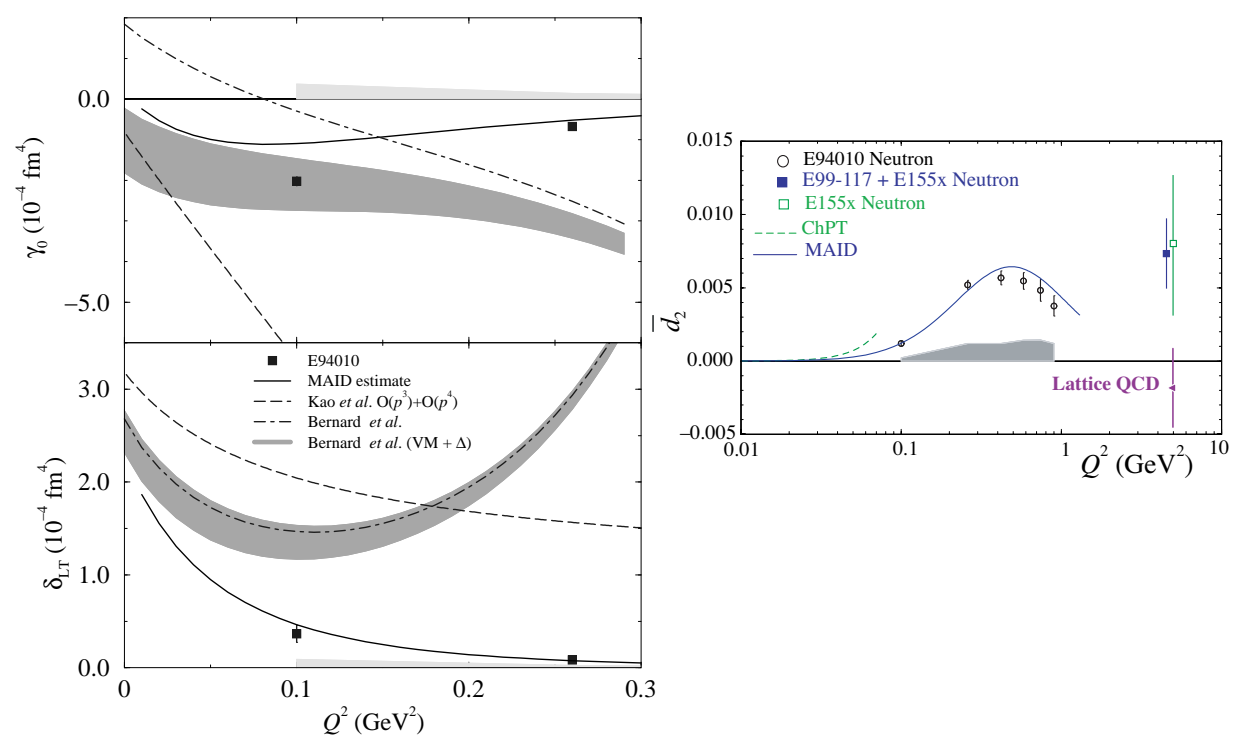

Fig. 3. Results for the neutron spin polarizabilities $\gamma_{0}$ (top-left panel) and $\delta_{L T}$ (bottomleft panel). Solid squares represent the results with statistical uncertainties. The light bands represent the systematic uncertainties. The dashed curves represent the HB $\chi \mathrm{PT}$ calculation. ${ }^{32}$ The dot-dashed curves and the dark bands represent the RB $\chi \mathrm{PT}$ calculation without and with ${ }^{24}$ the $\Delta$ and vector meson contributions, respectively. Solid curves represent the MAID model. ${ }^{3}$ The right panel shows the $\bar{d}_{2}^{n}$ results from $\mathrm{JLab}^{4,34}$ and SLAC, ${ }^{17}$ together with the Lattice QCD calculations. ${ }^{33}$

MAID model ${ }^{3}$ are also shown. At the lowest $Q^{2}$ point, the $\mathrm{RB} \chi \mathrm{PT}$ calculation including the resonance contributions is in good agreement with the experimental result. For the $\mathrm{HB} \chi \mathrm{PT}$ calculation without explicit resonance contributions, discrepancies are large even at $Q^{2}=0.1 \mathrm{GeV}^{2}$. This might indicate the significance of the resonance contributions or a problem with the heavy baryon approximation at this $Q^{2}$. The higher $Q^{2}$ data point is in good agreement with the MAID prediction, but the lowest data point at $Q^{2}=0.1 \mathrm{GeV}^{2}$ is significantly lower. Since $\delta_{L T}$ is insensitive to the $\Delta$ resonance contribution, it was believed that $\delta_{L T}$ should be more suitable than $\gamma_{0}$ to serve as a testing ground for the chiral dynamics of QCD. ${ }^{24,32}$ The bottom-left panel of Fig. 3 shows $\delta_{L T}$ compared to $\chi \mathrm{PT}$ calculations and the MAID predictions. While the MAID predictions are in good agreement with the results, it is surprising to see that the data are in significant disagreement with the $\chi \mathrm{PT}$ calculations even at the lowest $Q^{2}, 0.1 \mathrm{GeV}^{2}$. This disagreement presents a significant challenge to the present Chiral Pertubation Theory.

New experimental data have been taken at very low $Q^{2}$, down to 0.02 
$\mathrm{GeV}^{2}$ for the neutron $\left({ }^{3} \mathrm{He}\right)^{27}$ and the proton and deuteron. ${ }^{28}$ Analyses are underway. Preliminary asymmetry results just became available for the neutron. These results will shed light and provide benchmark tests to the $\chi \mathrm{PT}$ calculations at the kinematics where they are expected to work.

Another combination of the second moments, $d_{2}\left(Q^{2}\right)$, provides an efficient way to study the high $Q^{2}$ behavior of the nucleon spin structure, since it is a matrix element, related to the color polarizabilities and can be calculated from Lattice QCD. It also provides a means to study the transition from high to low $Q^{2}$. In Fig. $3, \bar{d}_{2}\left(Q^{2}\right)$ is shown. The experimental results are the solid circles. The grey band represents the systematic uncertainty. The world neutron results from SLAC ${ }^{17}$ (open square) and from JLab E99$117^{34}$ (solid square) are also shown. The solid line is the MAID calculation containing only the resonance contribution. At low $Q^{2}$ the $\mathrm{HB} \chi \mathrm{PT}$ calculation $^{32}$ (dashed line) is shown. The $\mathrm{RB} \chi \mathrm{PT}$ with or without the vector mesons and the $\Delta$ contributions ${ }^{24}$ are very close to the $\mathrm{HB} \chi \mathrm{PT}$ curve at this scale, and are not shown on the figure for clarity. The Lattice QCD prediction $^{33}$ at $Q^{2}=5 \mathrm{GeV}^{2}$ is negative but close to zero. There is a $2 \sigma$ deviation from the experimental result. We note that all models (not shown at this scale) predict a negative or zero value at large $Q^{2}$. At moderate $Q^{2}$, our data show that $\bar{d}_{2}^{n}$ is positive and decreases with $Q^{2}$.

Preliminary results at a $Q^{2}$ range of $1-4 \mathrm{GeV}^{2}$ for the neutron ${ }^{29}$ are available now. New experiments are planned with $6 \mathrm{GeV}$ beam ${ }^{30}$ at average $Q^{2}$ of $3 \mathrm{GeV}^{2}$ and with future $12 \mathrm{GeV}$ upgraded $\mathrm{JLab}^{31}$ at constant $Q^{2}$ values of 3,4 and $5 \mathrm{GeV}^{2}$. They will provide a benchmark test of the lattice QCD calculations.

\section{Conclusion}

A large body of nucleon spin-dependent cross-section and asymmetry data have been collected at low to moderate $Q^{2}$ in the resonance region. These data have been used to evaluate the $Q^{2}$ evolution of moments of the nucleon spin structure functions $g_{1}$ and $g_{2}$, including the GDH integral, the Bjorken sum, the BC sum and the spin polarizabilities.

At $Q^{2}$ close to zero, available next-to-leading order $\chi \mathrm{PT}$ calculations were tested against the data and found to be in reasonable agreement for $Q^{2}$ of 0.05 to $0.1 \mathrm{GeV}^{2}$ for the GDH integral $I\left(Q^{2}\right), \Gamma_{1}\left(Q^{2}\right)$ and the forward spin polarizability $\gamma_{0}\left(Q^{2}\right)$. Above $Q^{2}$ of $0.1 \mathrm{GeV}^{2}$ a significant difference between the calculation and the data is observed, pointing to the limit of applicability of $\chi \mathrm{PT}$ as $Q^{2}$ becomes larger. Although it was expected that the $\chi \mathrm{PT}$ calculation of $\delta_{L T}$ would offer a faster convergence because of 
the absence of the $\Delta$ contribution, the experimental data show otherwise. None of the available $\chi \mathrm{PT}$ calculations can reproduce $\delta_{L T}$ at $Q^{2}$ of 0.1 $\mathrm{GeV}^{2}$. This discrepancy presents a significant challenge to our theoretical understanding at its present level of approximations.

Overall, the trend of the data is well described by phenomenological models. The dramatic $Q^{2}$ evolution of $I_{G D H}$ from high to low $Q^{2}$ was observed as predicted by these models for both the proton and the neutron. This behavior is mainly determined by the relative strength and sign of the $\Delta$ resonance compared to that of higher-energy resonances and deep inelastic processes. This also shows that the current level of phenomenological understanding of the resonance spin structure using these moments as observables is reasonable.

The $\mathrm{BC}$ sum rule for both the neutron and ${ }^{3} \mathrm{He}$ is observed to be satisfied within uncertainties due to a cancellation between the resonance and the elastic contributions. The BC sum rule is expected to be valid at all $Q^{2}$. This test validates the assumptions going into the BC sum rule, which provides confidence in sum rules with similar assumptions.

Overall, the recent JLab data have provided valuable information on the transition between the non-perturbative to the perturbative regime of QCD. They form a precise data set for a check of $\chi \mathrm{PT}$ calculations. New results at very low $Q^{2}$ for the neutron, ${ }^{27}$ proton and deuteron ${ }^{28}$ will be available soon. They will provide benchmark tests of the Chiral Pertubation Theory calculations in the kinematical region where they are expected to work.

Future precision measurements ${ }^{30,31}$ of $d_{2}^{n}$ at $Q^{2}=3-5 \mathrm{GeV}^{2}$ will provide a benchmark test of Lattice QCD.

\section{Acknowledgments}

This work was supported by the U.S. Department of Energy (DOE). The Southeastern Universities Research Association operates the Thomas Jefferson National Accelerator Facility for the DOE under contract DE-AC0584ER40150, Modification No. 175.

\section{References}

1. see, e.g., B. W. Filippone and X. Ji, Adv. Nucl. Phys. 26, 1 (2001).

2. J. D. Bjorken, Phys. Rev. 148, 1467 (1966).

3. D. Drechsel, S. S. Kamalov and L. Tiator, Phys. Rev. D 63, 114010 (2001).

4. M. Amarian et al., Phys. Rev. Lett. 89, 242301 (2002);92, 022301 (2004);93, 152301 (2004); K. Slifer, et al., to be submitted to Phys. Rev. Lett.

5. R. Fatemi et al., Phys. Rev. Lett. 91, 222002 (2003). 
6. J. Yun et al., Phys. Rev. C 67, 055204 (2003).

7. A. Deur et al., Phys. Rev. Lett. 93, 212001 (2004).

8. K.V. Dharmawardane et al., Phys. Lett. B 64111 (2006); Y. Prok et al., to be published.

9. J.-P. Chen, A. Deur and Z.-E. Meziani, Mod. Phys. Lett. A 20, 2745 (2005).

10. S. B. Gerasimov, Sov. J. Nucl. Phys. 2, 598 (1965); S. D. Drell and A. C. Hearn, Phys. Rev. Lett. 16, 908 (1966).

11. X. Ji and J. Osborne, J. of Phys. G 27, 127 (2001).

12. D. Drechsel, B. Pasquini and M. Vanderhaeghen, Phys. Rep. 378, 99 (2003);

D. Drechsel and L. Tiator, Ann. Rev. Nucl. Part. Sci. 54, 69 (2004).

13. H. Burkhardt and W. N. Cottingham, Ann. Phys. (N.Y.) 56, 453 (1970).

14. X. Ji and P. Unrau, Phys. Lett. B 333, 228 (1994).

15. S. Wandzura and F. Wilczek, Phys. Lett. B 72, 195 (1977).

16. K. Ackerstaff et al., Phys. Lett. B 404, 383 (1997); B 444, 531 (1998).

17. K. Abe et al., Phys. Rev. D 58,112003 (1998); P. L. Anthony, et al., Phys. Lett. B 493, 19 (2000), B 553, 18 (2003).

18. J. Alcorn et al., Nucl. Inst. Meth. A522, 294 (2004).

19. B. A. Mecking et al., Nucl. Inst. Meth. A503, 513 (2003).

20. C. Ciofi degli Atti and S. Scopetta, Phys. Lett. B 404, 223 (1997).

21. Y. Liang et al., nucl-ex/0410027.

22. N. Bianchi and E. Thomas, Nucl. Phys. B 82 (Proc. Suppl.), 256 (2000).

23. X. Ji, C. Kao, and J. Osborne, Phys. Lett. B 472, 1 (2000).

24. V. Bernard, T. Hemmert and Ulf-G. Meissner, Phys. Lett. B 545, 105 (2002); Phys. Rev. D 67, 076008 (2003).

25. J. Soffer and O. V. Teryaev, Phys. Rev. D 70, 116004 (2004).

26. V. D. Burkert and B. L. Ioffe, Phys. Lett. B 296, 223 (1992).

27. JLab experiment E97-110, J. P. Chen, A. Deur, F. Garibaldi, spokespersons.

28. JLab run group eg4, M. Battaglieri, R. De Vita, A. Deur, M. Ripani spokespersons.

29. JLab E01-012, J. P. Chen, S. Choi and N. Liyanage, spokespersons.

30. JLab experiment E06-014, S. Choi, X. Jiang, Z.E. Meziani and B. Sawatzky spokespersons.

31. JLab experiment E12-06-121, T. Averett, W. Korsch, Z.E. Meziani and B. Sawatzky, spokespersons.

32. C. W. Kao, T. Spitzenberg and M. Vanderhaeghen, Phys. Rev. D 67, 016001 (2003).

33. M. Gockeler et al., Phys. Rev. D 63, 074506, (2001).

34. X. Zheng et al., Phys. Rev. C 70, 065207 (2004). 